\title{
Genetic polymorphism of epoxide hydrolase and glutathione S-transferase in COPD
}

\author{
S-L. Cheng*,\#, C-J. Yü, C-J. Chen", P-C. Yang"
}

Genetic polymorphism of epoxide hydrolase and glutathione $S$-transferase in COPD. S-L. Cheng, C-J. Yu, C-J. Chen, P-C. Yang. (C) ERS Journals Ltd 2004.

ABSTRACT: Genetic susceptibility to the development of chronic obstructive pulmonary disease (COPD) might depend on variation in the activities of enzymes that detoxify cigarette smoke products, such as microsomal epoxide hydrolase (mEPHX) and glutathione $S$-transferase (GST). It was investigated whether polymorphisms in these genes had any association with susceptibility to COPD and COPD severity.

The genotypes of 184 patients with COPD and 212 control subjects were determined by polymerase chain reaction followed by restriction fragment length polymorphism analysis of the mEPHX, GSTM1, GSTT1 and GSTP1 genes. All subjects were smokers or exsmokers.

The proportion of GSTM1-null genotypes was significantly higher in patients with COPD than in control subjects $(61.4$ versus $42.5 \%)$. No differences were observed in the frequency of polymorphic genotypes for mEPHX, GSTT1 and GSTP1. During combined analysis of genetic polymorphisms for mEPHX, GSTM1 and GSTP1, it was found that there are strong indicators for susceptibility to COPD (genotype combination with at least one mutant mEPHX exon-3 allele (histidine 113), GSTM1 null and homozygous for the GSTP1 isoleucine 105 allele). The frequencies of homozygous mutant alleles of mEPHX exon 3 and the GSTM1-null genotype were significantly higher in patients with severe COPD (forced expiratory volume in one second of $<35 \%$ of the predicted value).

It is proposed that the combination of genetic variants including at least one mutant microsomal epoxide hydrolase exon-3 allele and glutathione $S$-transferase M1-null and homozygous isoleucine 105 glutathione $S$-transferase P1 genotypes are significant indicators of susceptibility to chronic obstructive pulmonary disease in the Taiwanese population. In addition, the homozygous variant of microsomal epoxide hydrolase exon 3 and the glutathione $\boldsymbol{S}$-transferase M1-null genotype are independent risk factors for developing severe chronic obstructive pulmonary disease.

Eur Respir J 2004; 23: 818-824.
*Dept of Internal Medicine, Far Eastern Memorial Hospital, ${ }^{\#}$ Dept of Internal Mediçine, National Taiwan University Hospital and Graduate Institute of Epidemiology, College of Public Health, National Taiwan University, Taipei, Taiwan.

Correspondence: C-J. Yu

Dept of Internal Medicine

National Taiwan University Hospital

No. 7, Chung-Shan South Road

Taipei

Taiwan

Fax: 886223585867

E-mail: Jeffery@ha.mc.ntu.edu.tw

Keywords: Chronic obstructive pulmonary disease

epoxide hydrolase

glutathione $S$-transferase

polymorphism

Received: September 152003

Accepted after revision: January 282004
It is generally accepted that cigarette smoking is the most important risk factor for chronic obstructive pulmonary disease (COPD). Nevertheless, only $10-20 \%$ of chronic smokers develop the severe impairment of pulmonary function associated with COPD $[1,2]$. This indicates the possible contribution of environmental or genetic cofactors to the development of COPD. Although cofactors, such as childhood viral infection and environmental and occupational pollution, play important roles in pathogenesis [3], genetic susceptibility may be a factor of major importance [4].

The only established genetic risk factor for COPD is homozygosity of the $\mathrm{Z}$ allele of the $\alpha_{1}$-antitrypsin $\left(\alpha_{1}\right.$-AT) gene. Patients with genetic $\alpha_{1}$-AT deficiency have a very high risk of developing emphysema at an early age if they smoke. However, these patients account for only a small proportion of all patients with emphysema [5]. Recent studies reported that genetic variations in the enzymes that detoxify cigarette smoke products might be associated with the development of COPD. These enzymes include microsomal epoxide hydrolase (mEPHX), glutathione $S$-transferase (GST), and cytochrome $\mathrm{p}_{450}$ 1A1. mEPHX is an enzyme involved in the first-pass metabolism of smoking-induced highly reactive epoxide intermediates and is expressed at varying levels in most tissue and cell types [6, 7]. The human mEPHX gene is localised to the long arm of chromosome 1 [8], and two common aberrant alleles can be detected, which confer slow and fast enzyme activity [9]. An exon-3 thymine (T) to cytosine (C) mutation changes tyrosine residue 113 to histidine, and enzyme activity is reduced by $\geqslant 50 \%$ (slow allele). The second mutation, an adenine (A) to guanine (G) transition in exon 4 of the gene, changes histidine residue 139 to arginine, and produces an enzyme with an activity increased by $\geqslant 25 \%$ (fast allele). The distance between exon 3 and exon 4 is 6,696 base pairs [10].

GSTs are a superfamily of enzymes involved in the conjugation of a wide range of electrophilic substances with glutathione, thereby facilitating detoxification and further metabolism and excretion. GSTs are separated into the following classes: alpha, mu (GSTM), pi (GSTP), theta (GSTT), sigma and kappa. The GST M1, T1 and P1 genes are located on chromosomes $1 \mathrm{p} 13,22 \mathrm{q} 11.2$ and $11 \mathrm{q} 13$, respectively. Among the isoenzymes of GST, the homozygous GSTM1-null genotype has been reported to show some association with the pathogenesis of lung cancer [11, 12], bladder cancer [13] and, especially, emphysema [14]. The GSTT1-null mutant has also been suggested as a risk factor in 
many diseases [15-17]. Polymorphisms of GSTP1 genotypes have been reported, including isoleucine (Ile) 105 to valine (Val) mutation in exon 5 and alanine 114 to Val (Val) mutation in exon 6 . Individuals with the $\mathrm{Val}^{105}$ (mutant) allele have a higher risk of developing lung cancer than those with the Ile $^{105}$ (wild-type) allele [18]. In addition, GSTP1 is expressed more abundantly in respiratory tissues than other kinds of GST [19].

Previous evidence indicates that susceptibility to COPD is not a single-gene event. Moreover, ethnic differences exist. For example, COPD is uncommon in Chinese living in the USA, and the prevalence of Japanese-Americans smoking $>20$ cigarettes daily was much lower than in a matched Caucasian-American group (7.9 versus 16.7\%) [20]. Studies on familial clustering of COPD also demonstrated evidence of predominantly polygenic effects [21]. These findings all indicate that exploration of the risk factors for COPD at the molecular level will be informative and should be performed in various racial groups. Multiple genetic polymorphisms should be investigated to clarify whether the genetic events have additive effects or can predict the risk of developing COPD. In the present study, the relationship between the mEPHX, GSTM1, GSTT1 and GSTP1 genotypes and susceptibility to and severity of COPD in cigarette smokers in Taiwan was investigated.

\section{Patients and methods}

\section{Study population}

The study group consisted of 184 patients (152 males and 32 females) with smoking-related COPD recruited from the National Taiwan University Hospital (Taipei, Taiwan). COPD was diagnosed on the basis of medical history, chest radiographic findings, physical examination and spirometric data, according to American Thoracic Society (ATS) guidelines [1]. Inclusion criteria for COPD included the following: chronic airway symptoms and signs such as coughing, breathlessness, wheezing and chronic airway obstruction, defined as a forced expiratory volume in one second (FEV1)/ forced vital capacity (FVC) of $<70 \%$ and an FEV1 of $<80 \%$ of the predicted value from spirometric data; and FEV1 reversibility after inhalation of $200 \mu \mathrm{g}$ salbutamol of $<12 \%$ of prebronchodilator FEV1. Patients with COPD were classified into two subgroups according to severity (ATS criteria): mild/moderate COPD; and severe COPD, with an FEV1 of $<35 \%$ pred. Subjects were excluded if they had a history of asthma (reversibility of airflow obstruction) or malignant lung disease.

The control group included 212 asymptomatic smokers or exsmokers (182 males and 30 females) with a smoking history of $\geqslant 10$ pack-yrs without clinical or laboratory evidence of COPD. All were subjects who visited the National Taiwan University Hospital for a medical examination. All control subjects exhibited normal pulmonary function (FEV1/ FVC $>70 \%$ and FEV1 $>80 \%$ pred) [22]. Ethical approval and informed consent were obtained.

\section{DNA preparation}

Genomic deoxyribonucleic acid (DNA) was extracted from total blood cells using a QIAamp Blood MiniKit (QIAGEN, Hilden, Germany). Genomic DNA (20 ng) was amplified via polymerase chain reaction (PCR) using a thermal cycler (Minicycler $^{\mathrm{TM}}$, MJ Research, Inc., Waltham, MA, USA) in $40 \mu \mathrm{L}$ reaction mixture containing $1.5 \mathrm{mM} \mathrm{MgCl}_{2}, 100 \mathrm{ng}$ of each primer, $500 \mu \mathrm{M}$ deoxyribonucleoside triphosphate and 0.6 IU Taq DNA polymerase (MBI Fermentas, Hanover, MD, USA).

\section{PCR restriction fragment length polymorphism analysis} of microsomal epoxide hydrolase gene

The PCR conditions consisted of an initial single cycle of $10 \mathrm{~min}$ at $95^{\circ} \mathrm{C}$ followed by 35 cycles of $30 \mathrm{~s}$ at $94^{\circ} \mathrm{C}, 20 \mathrm{~s}$ at $52^{\circ} \mathrm{C}$ and $5 \mathrm{~s}$ at $72^{\circ} \mathrm{C}$. Specific mEPHX primers for the PCR-based genotyping assays were synthesised as follows: 5'-GATCGATAAGTTCCGTTTCACC-3' (exon 3, sense); 5'-ATCCTTAGTCTTGAAGTGAGGAT-3' (antisense; engineered base change, $\mathrm{G}$ to $\mathrm{A}$, underlined); 5'-ACATCCACTTCATCCACGT-3' (exon 4, sense); and 5'-ATGCCTCTGAGAAGCCAT-3' (antisense). The exon-4 mutation produces a Rhodopseudomonas sphaeroides (Rsa) I restriction fragment length polymorphism in the mutant (fast) allele (ATAC to GTAC). Each PCR product was digested to completion with Escherichia coli J62 pLG74 RV (exon 3) or Rsa I (exon 4) (New England BioLabs, Inc., Beverly, MA, USA), separated by electrophoresis through a $3 \%$ agarose gel, stained with ethidium bromide and transilluminated with ultraviolet light. The exon-3 wild-type allele was expected to yield 140- and 22-base-pair (bp) fragments, whereas the variant allele remained an uncleaved 162-bp fragment (fig.1a). Conversely, the exon-4 wild-type allele remained an uncleaved 210-bp fragment, whereas the variant allele was expected to yield 164- and 46-bp fragments (fig. 1b). According to the report of SMITH and HARRISON [23], the four groups of putative mEPHX phenotypes were classified as follows: normal (no mutation or heterozygous for both exon-3 and exon-4 mutations), fast (at least one fast mutation (exon 4) and no exon-3 mutations), slow (one slow (exon-3) mutant allele), and very slow (two slow alleles).

\section{Multiplex PCR amplification of glutathione S-transferase $M 1$ and $T 1$}

In order to detect deletion of the GST M1 and T1 genes, multiplex PCR was performed, using a $\beta$-globulin gene as an internal control, as described by CHEN et al. [16]. The primer pairs for each gene were as follows: GSTM1: 5'-GAACTCCCTGAAAAGCTAAAGC-3' and 5'-GTTGGGCTCAAATATACGGTGG-3'; GSTT1: 5'-TTCCTTACTGGTCCTCACATCTC-3' and 5'-CAGCTGCATTTGGAAG TGCTC-3'; and $\beta$-globulin: 5'-CAACTTCATCCACGTTCACC-3' and 5'-GAAGAGCCAAGGACAGGTAC-3'. The PCR buffer and cycles were the same as those used for mEPHX gene amplification. The products of the multiplex PCR (GSTM1 215 bp, GSTT1 480 bp and $\beta$-globulin 268 bp) were separated (fig. 1d). For both GST genes individually, subjects were categorised as having either a non-null or null (homozygous deletion) genotype.

$P C R$ restriction fragment length polymorphism analysis of glutathione S-transferase Pl gene

In the analysis of GSTP1 genotype, the exon-5 polymorphism (Ile ${ }^{105}$ to $\mathrm{Val}^{105}$ mutation) was chosen. The PCR and RFLP studies were performed using methods described by WATSON et al. [24] with a single modification. Assay of the exon-5 variant used the primer pairs 5'- GTAGTTTGCCCAAGGTCAAG-3' and 5'-AGCCACCTGAGGGGTAAG$3^{\prime}$. The PCR buffer and cycles were also the same as those 

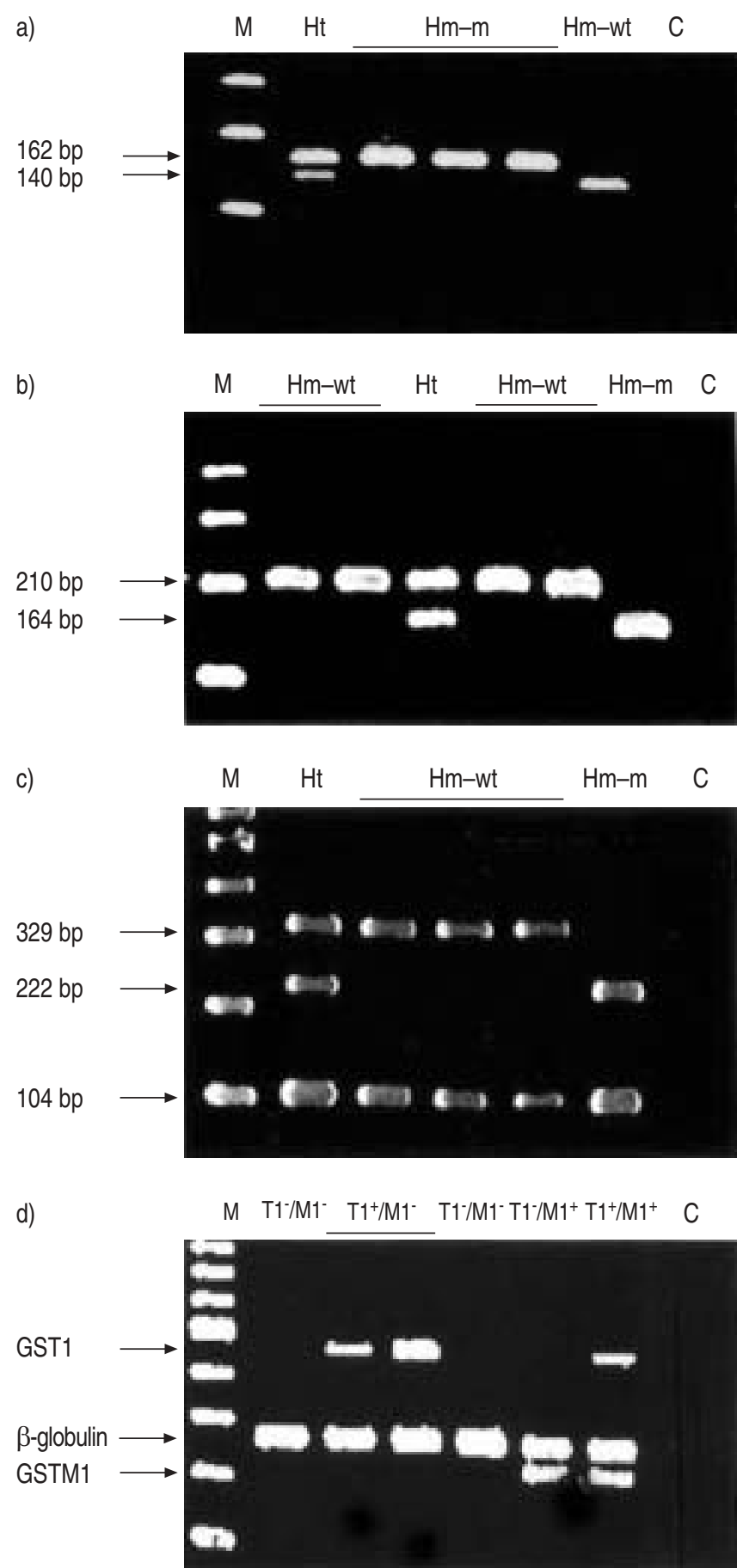

Fig. 1.- Polymerase chain reaction (PCR) restriction fragment length polymorphism analysis of: a) microsomal epoxide hydrolase (mEPHX) exon 3 ; b) mEPHX exon 4 ; c) glutathione $S$-transferase (GST) P1 gene; and d) multiplex PCR amplification of GST T1 (480 base pair (bp)) and M1 (215 bp) genes ( $\beta$-globulin internal control of 268 bp). M: 100-bp deoxyribonucleic acid ladder (marker); Ht: heterozygote; Hm: homozygote; m: mutant; wt: wild type; C: control (negative); $\mathrm{T}^{-}$: $\mathrm{T}^{-}$null; $\mathrm{M}^{-}$: $\mathrm{M}^{-}$null; $\mathrm{T}^{+}$: wt $\mathrm{T} 1$ gene; $\mathrm{M}^{+}$: wt $\mathrm{M} 1$ gene

used for mEPHX gene amplification. The PCR products were digested with 5 IU Bacillus stearothermophilus A664 AI (New England BioLabs, Inc.) for $1 \mathrm{~h}$ at $55^{\circ} \mathrm{C}$. The gel electrophoretic bands (wild-type 329 and $104 \mathrm{bp}$; mutant 222 and $104 \mathrm{bp}$ ) are shown in figure 1c.

\section{Statistical analysis}

Age, cumulative cigarette consumption and pulmonary function data are expressed as mean \pm SD. Statistical analysis of the relationship between genotype and clinical features was carried out using the Chi-squared or Fisher's exact test, as appropriate. A p-value of $<0.05$ was taken as significant. Hardy-Weinberg equilibrium was tested for all polymorphisms and no obvious deviation was found. The logistic regression model was used to calculate odds ratios and adjusted for age, sex and cumulative cigarette consumption between smokers with and without COPD. The mEPHX and GST assays place individuals into distinct categories: those not carrying or carrying at least one slow mEPHX allele, those with null or non-null GSTM1 and GSTT1 genotypes, and those with homozygous $\mathrm{Val}^{105}$ or heterozygous or homozygous $\mathrm{Ile}^{105}$ GSTP1 alleles. Odds ratios were also estimated in a referent group of individuals without any mEPHX slow alleles and with non-null GST M1 and T1 and homozygous $\mathrm{Val}^{105} / \mathrm{Val}^{105}$ GSTP1 genotypes.

\section{Results}

The age, sex, smoking history and pulmonary function data of patients with COPD and control subjects are summarised in table 1. No significant differences were observed in age or smoking history between patients and the control group. Hardy-Weinberg equilibrium was tested for all polymorphisms and no obvious deviation was found.

On multivariate analysis of the four genes, the frequency of the GSTM1-null genotype was significantly higher in the patients with COPD than in the control subjects (61.4 versus $42.5 \%$ ) (table 2$)$. The odds ratio was 2.2 (95\% confidence interval (CI) 1.3-3.5). The genotype frequencies of homozygous mutant mEPHX exon 3 (His ${ }^{113} / \mathrm{His}^{113}$ ) and $4\left(\mathrm{Arg}^{139} /\right.$ $\operatorname{Arg}^{139}$ ), GSTT1 null and wild-type GSTP1 (Ile ${ }^{105} / \mathrm{Ile}^{105}$ ) did not show significant difference between groups (table 2).

The mEPHX, GSTT1, GSTM1 and GSTP1 genotypes were analysed in combination in order to investigate whether a combination of these genetic polymorphisms was associated with the development of COPD. If the mEPHX genotype was classified into two subgroups of normal or fast and slow or very slow, there was a significant difference in mEPHX genotype between patients with COPD and controls (OR for slow/very slow versus normal/fast $2.3 ; 95 \%$ CI $1.1-4.3)$. The combined genotype of at least one slow mEPHX allele and GSTM1-null genotype occurred significantly more frequently in COPD patients than controls (48.4 versus $22.2 \%$ ). The odds ratio was 3.6 (95\% CI 1.9-8.6) compared to genotypes with normal and fast mEPHX alleles or the non-null GSTM1 genotype. The frequency in COPD patients was even higher

Table 1.-Age, sex, smoking and pulmonary function data of patients with COPD and controls

\begin{tabular}{lcc}
\hline & COPD & Controls \\
\hline Subjects n & 184 & 212 \\
Age yrs & $71.9 \pm 8.0^{\#}$ & $69.2 \pm 8.0$ \\
Males/females & $152 / 32$ & $182 / 30$ \\
Smoking pack-yrs & $36.6 \pm 11.5^{\star}$ & $33.2 \pm 12.8$ \\
FEV1 \% pred & $47.2 \pm 16.3^{* * *}$ & $103.2 \pm 13.7$ \\
FEV1/FVC & $45.8 \pm 8.6^{* * *}$ & $79.2 \pm 5.9$ \\
\hline
\end{tabular}

Data are presented as mean \pm SD. FEV1: forced expiratory volume in one second; FVC: forced vital capacity; \% pred: percentage of the predicted value. ${ }^{\#}: \mathrm{p}=0.23 ;{ }^{\bullet}: \mathrm{p}=0.12 ; * * *: \mathrm{p}<0.001$. 
Table 2.-Distribution of COPD associated with selected genetic polymorphisms of xenobiotic-metabolising enzymes

\begin{tabular}{|c|c|c|c|}
\hline Polymorphism & COPD & Controls & $\mathrm{OR}^{\#}(95 \% \mathrm{CI})$ \\
\hline Subjects n & 184 & 212 & \\
\hline \multicolumn{4}{|l|}{ mEPHX exon 3} \\
\hline $\operatorname{Tyr}^{113} / \operatorname{Tyr}^{113}$ & $33(17.9)$ & $56(26.4)$ & 1 \\
\hline $\operatorname{Tyr}^{113} / \mathrm{His}^{113}$ & $84(45.7)$ & $92(43.4)$ & $1.6(0.8-3.1)$ \\
\hline $\mathrm{His}^{113} / \mathrm{His}^{113}$ & $67(36.4)$ & $64(30.2)$ & $1.9(0.9-3.8)$ \\
\hline His $^{113}$ allele frequency & 0.59 & 0.48 & \\
\hline \multicolumn{4}{|l|}{ mEPHX exon 4} \\
\hline $\mathrm{His}^{139} / \mathrm{His}^{139}$ & $138(75.0)$ & $139(65.6)$ & 1 \\
\hline $\mathrm{His}^{139} / \mathrm{Arg}^{139}$ & $43(23.4)$ & $66(31.1)$ & $0.6(0.3-1.1)$ \\
\hline $\operatorname{Arg}^{139} / \mathrm{Arg}^{139}$ & $3(1.6)$ & $7(3.3)$ & $0.4(0.1-2.6)$ \\
\hline $\operatorname{Arg}^{139}$ allele frequency & 0.13 & 0.19 & \\
\hline \multicolumn{4}{|l|}{ GSTM1 } \\
\hline Non-null genotype & 71 (38.6) & $122(57.5)$ & 1 \\
\hline Null genotype & $113(61.4)$ & $90(42.5)$ & $2.2(1.3-3.5)^{\top}$ \\
\hline \multicolumn{4}{|l|}{ GSTT1 } \\
\hline Non-null genotype & $85(46.2)$ & $100(47.2)$ & 1 \\
\hline Null genotype & $99(53.8)$ & $112(52.8)$ & $0.9(0.6-1.6)$ \\
\hline \multicolumn{4}{|l|}{ GSTP1 } \\
\hline $\mathrm{Ile}^{105} / \mathrm{Ile}^{105}$ & $97(52.7)$ & $99(46.7)$ & $1.6(0.7-6.3)$ \\
\hline $\mathrm{Ile}^{105} / \mathrm{Val}^{105}$ & $78(42.4)$ & $98(46.2)$ & $1.2(0.4-3.9)$ \\
\hline $\mathrm{Val}^{105} / \mathrm{Val}^{105}$ & $9(4.9)$ & $15(7.1)$ & 1 \\
\hline $\mathrm{Ile}^{105}$ allele frequency & 0.74 & 0.64 & \\
\hline
\end{tabular}

Data are presented as n (\%) unless otherwise indicated. OR: odds ratio; CI: confidence interval; mEPHX: microsomal epoxide hydrolase; Tyr: tyrosine; His: histidine; Arg: arginine; GST: glutathione $S$-transferase; Ile: isoleucine; Val; valine. \#: adjusted for age, sex and cumulative cigarette consumption; ${ }^{\circ}: \mathrm{p}=0.004$.

compared to controls (36.4 versus $8.5 \%$ ) when the combined genotype was extended to include the homozygous wild-type $\left(\mathrm{Ile}^{105} / \mathrm{Ile}^{105}\right)$ GSTP1 genotype, and the odds ratio increased to 6.8 (95\% CI 1.6-17.7) (table 3).

Among the 184 patients with COPD, the severity of the COPD was classified as mild/moderate (FEV $1 \geqslant 35 \%$ pred) in 119 patients and severe (FEV1 $<35 \%$ pred) in 65 patients according to ATS guidelines [3]. There were no significant differences in smoking history between mild/moderate and severe patients $(31.2 \pm 2.9$ versus $35.9 \pm 2.6$ pack-yrs; $\mathrm{p}=0.22)$. The frequency of the homozygous $\mathrm{His}^{113} / \mathrm{His}^{113} \mathrm{mEPHX}$ exon-3 mutant was significantly higher in patients with severe COPD than in those with mild/moderate disease (63.1 versus $26.1 \%)$. The odds ratio was 7.5 (95\% CI 2.1-26.3) for the development of severe COPD, compared with patients with homozygous wild-type exon-3 alleles. No significant difference was observed when comparing the mutant mEPHX

Table 3. - Distribution of combinations of microsomal epoxide hydrolase (mEPHX) and glutathione $S$-transferase (GST) M1 and $\mathrm{P} 1$ genes among COPD patients and controls

\begin{tabular}{lccc}
\hline Genetic factors $^{*} \mathrm{n}$ & COPD & Controls & OR $^{\bullet}(95 \% \mathrm{CI})$ \\
\hline Subjects $\mathrm{n}$ & 184 & 212 & \\
$0^{+}$ & $52(28.3)$ & $93(43.9)$ & 1 \\
1 & $137(74.5)$ & $125(59.0)$ & $2.3(1.1-4.3)^{* * *}$ \\
2 & $89(48.4)$ & $47(22.2)$ & $3.6(1.9-8.6)^{* * *}$ \\
3 & $67(36.4)$ & $18(8.5)$ & $6.8(1.6-17.7)^{* * *}$ \\
\hline
\end{tabular}

Data are presented as $\mathrm{n}(\%)$ unless otherwise indicated. OR: odds ratio; CI: confidence interval. \#: $\geqslant 1$ mEPHX exon 3 slow allele, GSTM1-null genotype and $\mathrm{Ile}^{105} / \mathrm{Ile}^{105}$ GSTP1 added in sequence; ${ }^{\top}$ : adjusted for age, sex and cumulative cigarette consumption; ${ }^{+}$: reference type: no mEPHX exon 3 slow alleles, non-null GST M1 and T1 genotype and $\mathrm{Val}^{105} / \mathrm{Val}^{105}$ GSTP1; ***: p $<0.001$. exon- 4 allele frequency in the two groups of patients. Linkage disequilibrium was estimated between the two single nucleotide polymorphisms of mEPHX and no significant deviation was found $(\mathrm{p}=0.49)$. However, it cannot be concluded that exon 3 and exon 4 were in linkage equilibrium as only a very low number of mutant exon-4 homozygotes could be detected. As for GSTs, the distributions of GSTT1 and GSTP1 genotypes were not associated with COPD severity. However, there were significant differences in the distribution of GSTM1 genotypes and COPD severity. GSTM1-null genotypes occurred significantly more frequently than nonnull genotypes in patients with severe COPD (84.6 versus $52.9 \%$ ) with an odds ratio of 5.6 (95\% CI 2.2-13.9) (table 4).

\section{Discussion}

In a complex polygenic disease such as COPD, it is likely that the operation of multiple genes is necessary and that susceptibility to disease depends on the coincident actions of several genetic events due to polymorphisms. Polymorphism of each gene may impart only a small relative risk of COPD, and it is reasonable to speculate that the coexistence of several crucial polymorphisms is important in the pathogenesis of COPD. In the present study, mEPHX and GST M1, T1 and P1 genotypes were analysed in combination in order to elucidate the association between the various genotype combinations and development of COPD. The odds ratio was raised almost four-fold when the two genotypes of at least one slow mEPHX exon-3 allele and GSTM1 null were combined. The ratio increased to seven-fold with the addition of the homozygous $\mathrm{Ile}^{105} / \mathrm{Ile}^{105}$ GSTP1 genotype. It is

Table 4.-Analysis of the interaction between genetic polymorphisms of xenobiotic-metabolising enzymes and their association with COPD severity

\begin{tabular}{|c|c|c|c|}
\hline \multirow[t]{2}{*}{ Polymorphism } & \multicolumn{2}{|c|}{ FEV1 } & \multirow[t]{2}{*}{$\mathrm{OR}^{\#}(95 \% \mathrm{CI})$} \\
\hline & $<35 \%$ pred & $\geqslant 35 \%$ pred & \\
\hline Subjects $n$ & 65 & 119 & \\
\hline \multicolumn{4}{|l|}{ mEPHX exon 3} \\
\hline $\mathrm{Tyr}^{113} / \mathrm{Tyr}^{113}$ & $5(7.7)$ & $25(21.0)$ & 1 \\
\hline $\mathrm{Tyr}^{113} / \mathrm{His}^{113}$ & $19(29.2)$ & $63(52.9)$ & $1.9(0.5-7.0)$ \\
\hline $\mathrm{His}^{113} / \mathrm{His}^{113}$ & $41(63.1)$ & $31(26.1)$ & $7.5(2.1-26.3)^{* *}$ \\
\hline $\mathrm{His}^{113}$ allele frequency & 0.77 & 0.53 & \\
\hline \multicolumn{4}{|l|}{ mEPHX exon 4} \\
\hline $\mathrm{His}^{139} / \mathrm{His}^{139}$ & $46(70.8)$ & $91(76.5)$ & $0.9(0.1-7.8)$ \\
\hline $\mathrm{His}^{139} / \mathrm{Arg}^{139}$ & $17(26.2)$ & $27(22.7)$ & $0.8(0.1-4.2)$ \\
\hline Arg $^{139} /$ Arg $^{139}$ & $2(3.0)$ & $1(0.8)$ & 1 \\
\hline $\operatorname{Arg}^{139}$ allele frequency & 0.16 & 0.12 & \\
\hline \multicolumn{4}{|l|}{ GSTM1 } \\
\hline Non-null genotype & $10(15.4)$ & $56(47.1)$ & 1 \\
\hline Null genotype & $55(84.6)$ & $63(52.9)$ & $5.6(2.2-13.9)^{* *}$ \\
\hline \multicolumn{4}{|l|}{ GSTT1 } \\
\hline Non-null genotype & $32(49.2)$ & $53(44.5)$ & 1 \\
\hline Null genotype & $33(50.8)$ & $66(55.5)$ & $0.9(0.4-1.7)$ \\
\hline \multicolumn{4}{|l|}{ GSTP1 } \\
\hline $\mathrm{Ile}^{105} / \mathrm{Ile}^{105}$ & $32(49.2)$ & $64(53.8)$ & $0.6(0.1-2.4)$ \\
\hline $\mathrm{Ile}^{105} / \mathrm{Val}^{105}$ & $28(43.1)$ & $51(42.9)$ & $0.4(0.1-1.5)$ \\
\hline $\mathrm{Val}^{105} / \mathrm{Val}^{105}$ & $5(7.7)$ & $4(3.3)$ & 1 \\
\hline Ile $^{105}$ allele frequency & 0.70 & 0.75 & \\
\hline
\end{tabular}

Data are presented as $\mathrm{n}(\%)$ unless otherwise indicated. FEV1: forced expiratory volume in one second; \% pred: percentage of the predicted value; OR: odds ratio; $\mathrm{CI}$ : confidence interval; mEPHX: microsomal epoxide hydrolase; Tyr: tyrosine; His: histidine; Arg: arginine; GST: glutathione $S$-transferase; Ile: isoleucine; Val; valine; ${ }^{\#}$ : adjusted for age, sex and cumulative cigarette consumption; ${ }^{* *}: \mathrm{p}<0.01$. 
reasonable to expect that extended exposure to cigarette smoke pollution exposure leads to more severe destruction and inflammation of lung parenchyma in individuals with risk-conferring genetic polymorphisms. Therefore, the present study suggests that cigarette smokers carrying specific genetic polymorphisms of mEPHX, GSTM1 and GSTP1 are particularly susceptible to COPD.

Chronic tobacco smoking is a major risk factor in the development of COPD. However, only a relatively small proportion of smokers suffer from airway obstruction. Genetic factors are thought to be associated with this susceptibility. Genes involving protease/antiprotease and oxidant/antioxidant interactions are of special interest. In the current report, using a control group of smokers, the frequency of polymorphisms of three genes (at least one slow mEPHX exon-3 allele, GSTM1 null and homozygous $\mathrm{Il}^{105}$ / Ile $^{105}$ GSTP1) were obviously higher in patients with COPD than in controls after controlling for cumulative cigarette consumption. The chance of development of COPD in chronic heavy cigarette smokers increases stepwise as individuals carry from one to three genetic polymorphisms.

It should be acknowledged that multiple combined genotype comparisons have a number of drawbacks. Gene interactions must be considered. The present genes are situated on different chromosomes and linkage disequilibrium is not likely. However, each locus could be in linkage disequilibrium with an unknown casual gene(s). The potential for linkage disequilibrium is a fundamental limitation of the candidate gene association approach and depends on the linkage disequilibrium surrounding each locus in the study population. In addition, functional studies, including analysis of mEPHX and GST messenger ribonucleic acid and protein expression, should be continued to confirm the causal relationship before conclusions can definitely be drawn. Thirdly, multiple genotype comparisons of relatively small sample size may show bias and uncertainty. However, the present authors felt that there was still a significant trend towards association between these gene polymorphisms and the development of COPD.

It is known that allele frequencies vary between races. SMITH and HARRISON [23] reported first that the mEPHX gene polymorphism was associated with susceptibility to pulmonary emphysema. In their study, the mutant allele frequency of exon 3 was found to be higher in patients with pulmonary emphysema than in the control group (28 versus $6 \%$ ). However, no significant difference between COPD patients and controls in the mutant allele frequencies of mEPHX exon 3 or 4 could be demonstrated in two Japanese studies and one Korean study [25-27]. The distribution of mEPHX genotypes in the above three Asian studies are similar to those in the present study.

Unlike the mEPHX gene, the distribution patterns of GST genotypes are more complex among races. The frequency of the GSTT1-null genotype in the present control group $(52.8 \%)$ was close to that in the Korean population $(62 \%)$, but higher than in Caucasians (20.4\%) [27, 28]. Although the frequency of the GSTM1-null genotype in the present control group was similar to that found in Western countries (42.5 versus $46.9-53 \%$ ), it was lower than that found in Koreans $(65 \%)[14,18,27]$. The $\mathrm{Ile}^{105} / \mathrm{Ile}^{105}$ genotype distribution of GSTP1 in the present control subjects $(46.7 \%)$ was higher than in Westerners (35-42\%) [24], but lower than in Japanese $(52 \%)$ [29]. The difference in frequencies of genotype might be relevant to different metabolising enzyme activities and types of dominant functional enzymes against oxidative stress in different races.

In the present study, the frequencies of the GSTM1-null and homozygous wild-type (Ile $\mathrm{I}^{105} / \mathrm{Ile}^{105}$ ) GSTP1 genotypes were higher in patients with COPD. GSTP1 is expressed more abundantly than other GSTs in alveoli, alveolar macrophages and respiratory bronchioles [19], and is expected to be the major GST isoenzyme for local detoxification of xenobiotics in the lung. Wild-type GSTP1 shows weaker catalytic efficacy for carcinogenic aromatic epoxides than the Val ${ }^{105}$ GSTP1 mutant [30]. Therefore, GSTP1 isoenzymes from homozygous wild-type alleles exhibit less detoxifying capacity against xenobiotics in tobacco smoke. The null type of GSTM1 shows defective function in detoxifying the polycyclic aromatic hydrocarbons of cigarette smoke and promotes cellular and tissue damage of the lung due to an excess of oxidants and free radicals. Unlike GSTP1, an apparent decrease in or loss of GSTM1 expression in distal lung has been found in $56 \%$ of the population [19]. Thus the contribution of GSTM1 to the overall detoxifying capability of the lungs is controversial. However, several studies, including the current report, have documented the association of GSTM1 with susceptibility to lung diseases, such as lung cancer [11, 12] and COPD [14]. Therefore, the present authors speculate that people with the GSTM1-null and wild-type GSTP1 genotype tend to show a defective or weaker detoxifying capability in the lungs, as both GSTM1 and GSTP1 isoenzymes are more major catalytic enzymes against oxidative stress than other kinds of GST in the respiratory tract.

In patients with severe COPD (FEV1 $<35 \%$ pred), the frequency of mutant mEPHX exon-3 alleles and the GSTM1null genotype are significantly higher than in mild/moderate COPD. SANDFORD et al. [31] have recently reported the association of the homozygous $\mathrm{His}^{113} / \mathrm{His}^{139}$ (very slow) mEPHX genotype with rapid decline in lung function. Yoshikawa et al. [25] also considered individual homozygous variants of exon 3 to be associated with the development of advanced COPD, rather than susceptibility to COPD. The insufficient mEPHX enzyme activity may play an important role in the further progression of COPD disease status. The present findings are in agreement with the above studies and emphasise that patients with very severe airway obstruction (FEV1 $<35 \%$ pred) impairment show higher frequencies of homozygous variants of exon 3 than those with mild or moderate pulmonary function. The present study is also the first report documenting that the GSTM1-null genotype is an independent risk factor for developing severe COPD. The GSTM1-null genotype causes an obvious enzymatic defect of detoxifying function in the cellular defense against various toxic substances in tobacco smoke. Individuals with homozygous mEPHX exon 3 variants or the GSTM1-null genotype would show slow enzyme activity and may detoxify epoxides or other toxic particles in cigarette smoke less readily. Longterm exposure to xenobiotics would lead to greater tissue damage and inflammation of the lungs and leads to a more rapid decline in lung function. Thus it is reasonable to conclude that severe impairment of lung function may develop in individuals carrying the mutant mEPHX exon-3 allele, GSTM1-null genotype or both.

In the present study, several limitations remain. First, the numbers of males and females were not balanced and a relatively small population was recruited, especially in the severe COPD group. The major population of COPD patients and chronic smokers is male in Taiwan. Therefore, it is difficult to find a balanced set of female subjects for genotype studies. Secondly, the cases and control subjects were recruited from a tertiary university hospital. This kind of hospital-based recruitment of patients may cause selection bias. Thirdly, information regarding environmental pollution or inhaled irritants other than cigarette smoking is not available. Thus it is difficult to quantify their contribution to airway obstruction.

In summary, it was found that the coexistence of genetic variants, including at least one slow mutant microsomal epoxide hydrolase allele and the glutathione $S$-transferase 
M1-null (to give defective detoxifying enzymatic function) and homozygous isoleucine 105 glutathione $S$-transferase P1 (to exert less protective mechanism) genotypes, was a significant risk factor in susceptibility to chronic obstructive pulmonary disease in the Taiwanese population. Moreover, both the mutant allele of microsomal epoxide hydrolase exon 3 and the glutathione $S$-transferase M1-null genotype were independent factors for developing severe chronic obstructive pulmonary disease. In terms of the limitation in the number of subjects examined, the present study is a preliminary work and further studies should be performed using a larger population. Furthermore, the study of more candidate genes, such as those of xenobiotic phase I and II metabolising enzymes, remains necessary in order to elucidate the genetic pathogenesis of chronic obstructive pulmonary disease as a complex polygenic disease.

Acknowledgements. The authors would like to thank C.A.D. Smith and D.J. Harrison for technical assistance in the microsomal epoxide hydrolase polymorphism genotyping assays.

\section{References}

1. Fletcher C, Peto R, Tinker C, Speizer FE. The Natural History of Chronic Bronchitis and Emphysema: an EightYears Follow-Up Study of Working Men in London. Oxford, Oxford University Press, 1976; pp. 70-105.

2. Bascom R. Differential susceptibility to tobacco smoke: possible mechanisms. Pharmacogenetics 1991; 1: 102-106.

3. American Thoracic Society. Standards for the diagnosis and care of patients with chronic obstructive pulmonary disease. Am J Respir Crit Care Med 1995; 152: S77-S120.

4. Sandford AJ, Weir TD, Pare PD. Genetic risk factors for chronic obstructive pulmonary disease. Eur Respir J 1997; 10: $1360-1391$.

5. Kuppers F, Black LF. Alpha 1-antitrypsin and its deficiency. Am Rev Respir Dis 1974; 110: 176-194.

6. Oesch F. Mammalian epoxide hydrolase: inducible enzymes catalyzing the inactivation of carcinogenic and cytogenic metabolites derived from aromatic and olefinic compounds. Xenobiotica 1973; 3: 305-340.

7. Oesch F, Glatt H, Schmassmann H. The apparent ubiquity of epoxide hydratase in rat organs. Biochem Pharmacol 1977; 26: 603-607.

8. Skoda RC, Demierre A, McBride OW, Gonzalez FJ, Meyer UA. Human microsomal xenobiotic epoxide hydrolase. Complementary DNA sequence, complementary DNAdirected expression in COS-1 cells, and chromosomal localization. J Biol Chem 1988; 263: 1549-1554.

9. Hassett C, Aicher L, Sidhu JS, Omiecinski CJ. Human microsomal epoxide hydrolase: genetic polymorphism and functional expression in vitro of amino acid variants. Hum Mol Genet 1994; 3: 421-428.

10. Hassett C, Robinson KB, Beck NB, Omiecinski CJ. The human microsomal epoxide hydrolase gene (EPHX1): complete nucleotide sequence and structural characterization. Genomics 1994; 23: 433-442.

11. Seidegard J, Pero RW, Miller DG, Beattie EJ. A glutathione transferase in human leukocytes as a marker for the susceptibility to lung cancer. Carcinogenesis 1986; 7: 751753.

12. Hirvonen A, Husgafvel-Pursiainen K, Anttila S, Vainio H. The GSTM1 null genotype as a potential risk modifier for squamous cell carcinoma of the lung. Carcinogenesis 1993; 14: 1479-1481.

13. Bell DA, Taylor JA, Paulson DF, Robertson CN, Mohler
JL, Lucier GW. Genetic risk and carcinogen exposure: a common inherited defect of the carcinogen-metabolism gene glutathione $S$-transferase M1 that increases susceptibility to bladder cancer. $J$ Natl Cancer Inst 1993; 85: 11591164.

14. Harrison DJ, Cantlay AM, Rae F, Lamb D, Smith CAD. Frequency of glutathione $S$-transferase M1 deletion in smokers with emphysema and lung cancer. Hum Exp Toxicol 1997; 16: 356-360.

15. Chenevix-Trench G, Young J, Coggan M, Board P. Glutathione $S$-transferase M1 and T1 polymorphism: susceptibility to colon cancer and age of onset. Carcinogenesis 1995; 16: 1655-1657.

16. Chen H, Sandler DP, Tayor JA, et al. Increased risk for myelodysplastic syndrome in individuals with glutathione transferase theta 1 gene defect. Lancet 1996; 347: 295-297.

17. Elexpuru-Camiruaga $\mathrm{J}$, Buxton $\mathrm{N}$, Kandula $\mathrm{V}$, et al. Susceptibility to astrocytoma and meningioma: influence of allelism at glutathione $S$-transferase (GSTT1, GSTM1) and cytochrome P-450 (CYP2D6) loci. Cancer Res 1995; 55: 4237-4239.

18. Ryberg D, Skaug V, Hewer A, et al. Genotypes of glutathione transferase $\mathrm{M} 1$ and $\mathrm{P} 1$ and their significance for lung DNA adduct levels and cancer risk. Carcinogenesis 1997; 18: 1285-1289.

19. Cantlay AM, Smith CAD, Wallace WA, Yap P-L, Lamb D, Harrison DJ. Heterogeneous expression and polymorphic genotype of glutathione $S$-transferase in human lung. Thorax 1994; 49: 1010-1014.

20. Marcus EB, Buist AS, Curb JD, et al. Correlates of FEV1 and prevalence of pulmonary conditions in Japanese-American men. Am Rev Respir Dis 1988; 138: 1398-1404.

21. Givelber RJ, Courpmitree NN, Gottlieb DJ, et al. Segregation analysis of pulmonary function among families in the Framingham study. Am J Respir Crit Care Med 1998; 157: $1445-1451$.

22. Wu MC. Study on maximal expiratory flow and volume in Chinese. I. Normal nonsmoking adults. J Formos Med Assoc 1981; 80: 19-29.

23. Smith CAD, Harrison DJ. Association between polymorphism in gene for microsomal epoxide hydrolase and susceptibility to emphysema. Lancet 1997; 350: 630-633.

24. Watson MA, Stewart RK, Smith GBJ, Massey TE, Bell DA. Human glutathione $S$-transferase $\mathrm{P} 1$ polymorphisms: relationship to lung tissue enzyme activity and population frequency distribution. Carcinogenesis 1998; 19: 275-280.

25. Yoshikawa M, Hiyama K, Ishioka S, Maeda H, Maeda A, Yamakido M. Microsomal epoxide hydrolase genotypes and chronic obstructive pulmonary disease in Japanese. Int $\mathrm{J} \mathrm{Mol}$ Med 2000; 5: 49-53.

26. Takeyabu K, Yamaguchi E, Suzuki I, Nishimura M, Hizawa N, Kamakami Y. Gene polymorphism for microsomal epoxide hydrolase and susceptibility to emphysema in a Japanese population. Eur Respir J 2000; 15: 891894.

27. Yim JJ, Park GY, Lee CT, et al. Genetic susceptibility to chronic obstructive pulmonary disease in Koreans: combined analysis of polymorphic genotypes for microsomal epoxide hydrolase and glutathione $S$-transferase M1 and T1. Thorax 2000; 55: 121-125.

28. Nelson HH, Wiencke JK, Christiani DC, et al. Ethnic differences in the prevalence of the homozygous deleted genotype of glutathione $S$-transferase theta. Carcinogenesis 1995; 16: 1243-1245.

29. Ishii $\mathrm{T}$, Matsuse $\mathrm{T}$, Teramoto $\mathrm{S}$, et al. Glutathione $S$-transferase (GSTP1) polymorphism in patients with chronic obstructive pulmonary disease. Thorax 1999; 54: 693-696.

30. Sundberg K, Johansson AS, Stenberg G, et al. Differences in 
catalytic efficiencies of allelic variants of glutathione transferase P1-1 towards carcinogenic diol epoxides of polycyclic aromatic hydrocarbons. Carcinogenesis 1998; 19: 433-436.
31. Sandford AJ, Chagani T, Weir TD, Connett JE, Anthonisen NR, Pare PD. Susceptibility genes for rapid decline of lung function in the Lung Health Study. Am J Respir Crit Care Med 2001; 163: 469-473. 